USC-00/005, CITUSC/00-063

hep-th/0011207

\title{
Holographic Renormalization Group Flows: The View from Ten Dimensions 审
}

\author{
Nicholas P. Warner \\ Department of Physics and Astronomy, University of Southern California \\ Los Angeles, CA 90089-0484, USA \\ and \\ CIT-USC Center for Theoretical Physics \\ University of Southern California, Los Angeles, CA 90089-0484, USA
}

\begin{abstract}
The holographic description of supersymmetric RG flows in supergravity is considered from both the five-dimensional and ten-dimensional perspectives. An $\mathcal{N}=1^{*}$ flow of $\mathcal{N}=4$ super-Yang Mills is considered in detail, and the infra-red limit is studied in terms of IIB supergravity in ten dimensions. Depending on the vevs and the direction of approach to the core, the supergravity solution can be interpreted in terms of either 5-branes or 7-branes. Generally, it is shown that it is essential to use the ten-dimensional description in order to study the infra-red asymptotics in supergravity.
\end{abstract}

\section{Introduction}

Since its original formulation, the holographic AdS/CFT correspondence [1, [] has undergone some remarkable developments and extensions. In particular the holographic correspondence has been generalized to many non-conformal settings, and most particularly in the study of renormalization group (RG) flows. For the latter [3, [] one starts from a UV conformal fixed point field theory on a brane, represented by an AdS solution in supergravity. Fields on the brane then represent boundary data (at infinity in AdS) for a supergravity solution [2]: That is, one perturbs the theory on the brane and finds the corresponding supergravity solution with the boundary data at infinity determined by the perturbing fields. The renormalization group flow is then believed to be given by looking at the radial evolution of the supergravity solution in a direction transverse to the brane. The cosmological scale factor in front of the brane metric becomes the RG scale, and a generic, positive energy perturbation on the brane causes this cosmological factor to decrease with the radius [4]. Thus the deep interior of the supergravity solution probes the infra-red structure of the perturbed field theory.

$\dagger$ Talk presented at the Gürsey Memmorial Conference II, on M-theory and Dualities, held at Bogazici University, Istanbul, Turkey, June 19-23, 2000. 
In this paper I will consider supersymmetric flows generated by mass perturbations, and special vevs in $\mathcal{N}=4$ Yang-Mills, and in particular I will focus on the infra-red structure suggested by the dual supergravity solutions. There are several reasons for considering these particular flows. First, the duality between IIB supergravity and large $N, \mathcal{N}=4$ Yang-Mills on $D 3$-branes is perhaps the most extensively studied holographic correspondence and is therefore a natural starting point for studying non-conformal generalizations of the holographic correspondence. Secondly, supersymmetric flows afford the best oppurtunity to test the non-conformal, holographic correspondence. This is primarily because the supersymmetry, and associated non-renormalization theorems, enable the exact computation of some parts of the quantum effective action, or the determination of exact beta-functions [5] in the field theory on the brane. Moreover, the question of whether a supergravity calculation for large $N$ field theory has a well defined, finite $N$ counterpart is essentially the same as whether a solution to the supergravity equations represents a background "vacuum" for the complete string theory. One of the lessons of the last fifteen years has been that supersymmetric supergravity solutions can usually be generalized to string theory. In holographic language this means that supersymmetric supergravity solutions have a good chance of telling us something about finite $N$ field theories, while non-supersymmetric solutions are in danger of merely probing large $N$ pathologies.

While the D3-brane/IIB supergravity correspondence is framed in ten dimensions, it is possible to reduce one of the most important sectors down to a five-dimensional description. More precisely, gauged $\mathcal{N}=8$ supergravity in five dimensions provides an exact description of the "massless" sector of IIB supergravity reduced on $S^{5}$. In terms of the physics on the brane, this means that one can use $\mathcal{N}=8$ supergravity in five dimensions to study mass perturbations and special vevs in $\mathcal{N}=4$ supersymmetric Yang-Mills theory. The benefit of using the five-dimensional formalism is that the structure and equations of motion are far simpler than their ten-dimensional counterparts, and this has enabled extensive analysis of RG flows, and most particularly for the supersymmetric flows (see, for example [6, 4, 7, 8, 9, 10, 11]).

The reduction of the supergravity flow to a five-dimensional problem thus provides an extremely powerful computational tool, but there is a price: the correct physical interpretation of the flows is far from transparent in the five-dimensional world. Indeed, it is very easy to arrive at the wrong physical picture because of this five-dimensional "myopia". It is one of the primary purposes of this paper to show that the proper physical interpretation of the supergravity solutions requires that one find their tendimensional counterparts. It is important to stress that the five-dimensional solutions are neither wrong, nor incomplete, but that they encode the ten-dimensional data in a rather subtle manner that can obscure much of the physics. I will illustrate this in some detail for the holographic description of the $\mathcal{N}=1^{*}$ flows in which $\mathcal{N}=4$ Yang-Mills is broken to $\mathcal{N}=1$ by giving an equal mass to all the chiral multiplets [8, 12, 16]. However, the issues addressed here have far wider consequences, especially for attempted descriptions of field theory via five-dimensional "brane worlds" (see, for 
example, 17, 18]).

\section{Flows in five dimensions}

I will be considering the special class of fields in IIB supergravity that correspond to the "massless" perturbations of the $A d S_{5} \times S^{5}$ background. As is, by now, well known, this set of fields forms a consistent, closed subsector of the IIB supergravity theory, and the complete action for this subsector is given by that of gauged $\mathcal{N}=8$ supergravity in five dimensions [19. In particular it is believed (with very considerable evidence) that any field configuration of the five-dimensional theory can be embedded in the ten-dimensional theory, and that solutions to the five-dimensional equations of motion "lift" uniquely to solutions of the ten-dimensional theory. Thus working in the five-dimensional theory restricts the class of fields considered, but does not restrict the validity of the solution in ten dimensions.

The five-dimensional solutions corresponding to RG flows have 5-metrics of the form:

$$
d s^{2}=e^{2 A(r)} \eta_{\mu \nu} d x^{\mu} d x^{\nu}-d r^{2} .
$$

(I will adopt the conventions of [4] throughout.) This metric preserves Poincaré invariance on the D3-brane, but allows for a "cosmological" scale factor, and in the flow interpretation, $e^{A(r)}$ represents the RG scale on the branes. For the flow solutions, one requires that $A(r) \sim r / L$ as $r \rightarrow \infty$ so that the flow starts with the conformally invariant $A d S_{5}$ metric of radius $L$. One also usually only considers non-trivial scalar backgrounds that depend solely on $r$ in the five-dimensional supergravity since this also respects the Poincaré invariance on the branes. In $\mathcal{N}=8$ gauged supergravity, there are forty-two scalars, $\varphi_{j}$, and in the Yang-Mills theory these scalars are dual to the gauge coupling, the $\theta$-angle, the fermion bilinear operators and the scalar bilinear operators. The latter have the form:

$$
\operatorname{Tr}\left(\lambda^{a} \lambda^{b}\right), \quad \operatorname{Tr}\left(\bar{\lambda}^{\bar{a}} \bar{\lambda}^{\bar{b}}\right), \quad \operatorname{Tr}\left(X^{A} X^{B}\right)-\frac{1}{6} \delta^{A B} \operatorname{Tr}\left(X^{C} X^{C}\right) .
$$

In terms of representations of the $S O(6) \mathcal{R}$-symmetry, the foregoing operators transform as $\mathbf{1 0} \oplus \overline{\mathbf{1 0}} \oplus \mathbf{2 0}^{\prime}$.

The supergravity theory has a highly non-trivial scalar potential, $\mathcal{P}\left(\varphi_{i}\right)$, and the scalar equations of motion reduce to

$$
\frac{d^{2}}{d r^{2}} \varphi_{j}+4 A^{\prime}(r) \frac{d}{d r} \varphi_{j}-\frac{\partial \mathcal{P}}{\partial \varphi_{j}}=0
$$

For each scalar field there are generically two solutions to this equation, one of which represents a non-normalizable mode of $A d S_{5}$, and the other representing a normalizable mode. The former represents the insertion of a mass term, i.e. the insertion of one of the operators in (2) into the brane action, while the normalizable modes represent modifications to the state on the brane through operator vevs.

If one requires that the flow preserve at least one supersymmetry on the brane then

one must restrict how the scalars flow. In practice this is usually done by restricting 
to a subset of the scalars. One first shows that this restriction is consistent with the equations of motion, and then this restricted class of flows can usually be characterized in terms of a first order system:

$$
\frac{d \varphi_{j}}{d r}=\frac{g}{2} \frac{\partial W}{\partial \varphi_{j}}, \quad \text { and } \quad A^{\prime}(r)=-\frac{g}{3} W,
$$

where, on the restricted subsector, $W$ is a superpotential that is related to $\mathcal{P}$ via:

$$
\mathcal{P}=\frac{g^{2}}{8} \sum_{j=1}^{3}\left|\frac{\partial W}{\partial \varphi_{j}}\right|^{2}-\frac{g^{2}}{3}|W|^{2} .
$$

In these expressions, $g=2 / L$, is the coupling of the gauged supergravity, and $L$ is the radius of the asymptotic $A d S_{5}$ near infinity. Henceforth I shall normalize $L$ by setting $L=1$.

One can show very generally that $A^{\prime}(r)$ must increase monotonically as $r$ decreases [4], and it follows that $e^{2 A(r)}$ will go to zero as as $r$ decreases. There are thus two possibilities: either $e^{2 A(r)}$ vanishes only as $r \rightarrow-\infty$, or it vanishes at finite $r$ with

$$
e^{A(r)} \sim\left(r-r_{0}\right)^{2 a},
$$

for some exponent $a$. In terms of the superpotential, it can either flow to a critical point, or it can "flow to Hades:" $W \rightarrow-\infty$. The former represents a flow to a non-trivial, supersymmetric IR fixed point theory on the brane, and the geometry is non-singular, and interpretation is straightforward. An example of this was studied in detail in [4, 14].

Flows to Hades, in which $e^{A}$ behaves as in (6), appear to be singular as fivedimensional geometries, and one might be concerned that they are unphysical. However, there are quite a number of examples of physically sensible flows that have such asymptotics. The reason why we know that such flows are sensible is that we can lift them to ten dimensions, and analyse them in the IIB supergravity theory (see, for example, [7]). I will return to this in the next section. For the present I simply wish to note that the five-dimensional metric contains rather minimal information about the IR asymptotics: All one has is the exponent, $a$.

The flows that I particularly wish to study here are the $\mathcal{N}=1^{*}$ flows considered in [8]. On the brane the $\mathcal{N}=4$ Yang-Mills theory is broken to $\mathcal{N}=1$, large $\mathrm{N}$, "superQCD" by giving all three chiral multiplets a mass. For simplicity, these masses are taken to be equal, and the resulting theory preserves a global $S O(3)$ symmetry. In terms of fermion bilinears, this means that one is turning on a (complex) supergravity scalar, $m$, that is dual to:

$$
m \sum_{j=1}^{3} \operatorname{Tr}\left(\lambda^{j} \lambda^{j}\right)+\bar{m} \sum_{\bar{j}=1}^{3} \operatorname{Tr}\left(\bar{\lambda}^{\bar{j}} \bar{\lambda}^{j}\right) .
$$

While there is no explicit low energy supergravity field that is dual to the corresponding scalar mass term, $\sum_{A=1}^{6} \operatorname{Tr}\left(X^{A} X^{A}\right)$, the supergravity theory always implicitly adjusts the solution to contain the proper amount of this field as required by supersymmetry. One can also consider what happens if the gaugino condensate $\sigma \equiv \operatorname{Tr}\left(\lambda^{4} \lambda^{4}\right)$ is allowed to run simultaneously. 
Since they will play a major role later, it is important to understand the residual $\mathcal{R}$-symmetries of this scalar sub-sector. The original symmetry of the theory is $S O(6) \times S L(2, \mathbb{R})$, where the latter describes the scalar coset of the IIB supergravity. The $S L(2, \mathbb{R})$ is a symmetry of the supergravity, but it is broken to $S L(2, \mathbb{Z})$ in the string theory. In particular, the $U(1) \subset S L(2, \mathbb{R})$ represents "continuous duality symmetry" that must be broken in the string theory. In terms of the field theory on the brane, this "duality" $U(1)$ will be a property of the large $N$ theory, and will be broken at finite $N$. As remarked above, the mass perturbation preserves at least an $S O(3) \subset S O(6)$, which acts in the $S O(3)$ vector representation on the first three fermions $\lambda^{i}, i=1,2,3$. This $S O(3)$ also remains unbroken when $\sigma$ is turned on as well. The $S O(3)$ commutes with an $S O(2)$ inside $S O(6)$. Specifically, the generators of this $S O(3) \times S O(2)$ are the $6 \times 6$ matrices:

$$
\left(\begin{array}{cc}
J_{i} & 0 \\
0 & J_{i}
\end{array}\right), \quad\left(\begin{array}{cc}
0 & \mathbf{I}_{3 \times 3} \\
-\mathbf{I}_{3 \times 3} & 0
\end{array}\right) .
$$

This $S O(2)$ rotates $m$ and $\sigma$ by different phases. Any one, but not both, of these phase rotations can be undone by a $U(1)$ duality rotation. Hence turning on $m$ or $\sigma$ alone preserves an $S O(3) \times S O(2)$ 团, however, this extra $S O(2)$ rotation involves a $U(1)$ duality rotation and so will not be a symmetry of the finite $N$ field theory.

When both $m$ and $\sigma$ are turned on, the only remaining continuous symmetry is $S O(3)$. However, there is still residual discrete symmetry: Let $S=\left(\begin{array}{cc}0 & 1 \\ -1 & 0\end{array}\right) \in$ $S L(2, \mathbb{R})$, and define $R$ to be the discrete, $90^{\circ}$ rotation defined by taking the second matrix in (8) to be an element of the group $S O(6)$. The combination of these, $R S$, has order 4 , and is a symmetry of the flows for arbitrary $m$ and $\sigma$. Observe that $S$ is indeed the $S$-duality transformation in $S L(2, \mathbb{Z}) \subset S L(2, \mathbb{R})$, and indeed this should be a symmetry of the string theory. It follows that whatever structure one finds in the infra-red, one will find its $S$-dual at $90^{\circ}$ to that structure. This means that one can never see confinement in this class of supergravity solutions without fine tuning of the Wilson or 't Hooft loop: Any confining loop can always break to a screened loop by making a $90^{\circ}$ change of direction near the core of the solution.

The superpotential on this sector is:

$$
W=-\frac{3}{4}\left(\cosh (2 \sigma)+\cosh \left(\frac{2 m}{\sqrt{3}}\right)\right)
$$

where I have used the symmetries of the problem to take $m$ amd $\sigma$ to be real. The equations (4) then have solutions [8]:

$$
\begin{aligned}
& m=\frac{\sqrt{3}}{2} \log \left(\frac{1+t}{1-t}\right), \quad \sigma=\frac{1}{2} \log \left(\frac{1+\lambda t^{3}}{1-\lambda t^{3}}\right), \\
& A(r)=\frac{1}{6} \log \left(t^{-3}-\lambda^{2} t^{3}\right)+\frac{1}{2} \log \left(t^{-1}-t\right)+C_{1},
\end{aligned}
$$

where

$$
t=\exp \left[-\left(r-C_{1}\right)\right], \quad \lambda=\exp \left[3\left(C_{2}-C_{1}\right)\right],
$$

$\ddagger$ In fact, flows that involve $\sigma$ alone preserve an $S U(3) \times U(1)$. 
and where the $C_{j}$ are constants of integration for the flows of $m$ and $\sigma$. As noted in [8], the physical metrics should have $\lambda \leq 1$ since one should not have the gaugino condensate diverging before the mass scale diverges.

The five dimensional metric becomes:

$$
d s^{2}=-d r^{2}+\left(t^{-1}-t\right)\left(t^{-3}-\lambda^{2} t^{3}\right)^{\frac{1}{3}} \eta_{\mu \nu} d x^{\mu} d x^{\nu} .
$$

This is manifestly singular at $t=1$, and the exponent, $a$, is $\frac{1}{2}$ for $\lambda<1$, and $\frac{2}{3}$ for $\lambda=1$. The structure of this singularity elucidates very little of the physics. To go any further, it is necessary to reconstruct the ten-dimensional counterparts of these flows.

\section{The view from ten dimensions}

It was shown in [6, 15] that the metric and dilaton configuration in ten dimensions can be reconstructed from the scalar field configuration in five dimensions. Specifically, let $\left(\mathcal{V}^{I J}{ }_{a b}, \mathcal{V}_{I \alpha}{ }^{a b}\right)$ be the components of the $E_{6(6)}$ matrix that characterizes the scalar fields as in [19], and let $\left(\widetilde{\mathcal{V}}_{I J}{ }^{a b}, \tilde{\mathcal{V}}^{I \alpha}{ }_{a b}\right)$ be the inverse of $\mathcal{V}$. Let $K^{I J p}$ be the fifteen Killing vectors on the round $S^{5}$, and let $x^{J}$ denote the Cartesian coordinates of the standard embedding of $S^{5}$ in $\mathbb{R}^{6}$. Then the ten-dimensional metric has the form

$$
d s_{10}^{2}=\Delta^{-\frac{2}{3}} d s_{5}^{2}+d \tilde{s}_{5}^{2},
$$

where $d s_{5}^{2}$ is the metric, (西), of the five-dimensional theory, and $d \tilde{s}_{5}^{2}=\tilde{g}_{m n} d y^{m} d y^{n}$ is the metric on the deformed five-sphere. The inverse of this metric is then given by

$$
\Delta^{-\frac{2}{3}} \widetilde{g}^{p q}=\frac{1}{c^{2}} K^{I J p} K^{K L q} \widetilde{\mathcal{V}}_{I J a b} \widetilde{\mathcal{V}}_{K L c d} \Omega^{a c} \Omega^{b d}
$$

where $c$ is a normalization constant, and $\Omega^{a b}$ is a $U S p(8)$ symplectic form. The warpfactor, $\Delta$, is defined by

$$
\Delta \equiv \sqrt{\operatorname{det}\left(g_{m p} \stackrel{\circ}{g} p q\right)} .
$$

where the inverse metric, $\stackrel{\circ}{g} p q$, is that of the "round" $S^{5}$. One can determine $\Delta$ from (15) by taking the determinant on both sides.

Similarly, if $\mathcal{S} \in S L(2, \mathbb{R})$ describes the dilation/axion of the IIB theory, then the gauge independent quantity $\mathcal{S S}^{T}$ is given by

$$
\Delta^{-\frac{4}{3}}\left(\mathcal{S} \mathcal{S}^{T}\right)^{\alpha \beta}=\text { const } \times \epsilon^{\alpha \gamma} \epsilon^{\beta \delta} \mathcal{V}_{I \gamma}{ }^{a b} \mathcal{V}_{J \delta}{ }^{c d} x^{I} x^{J} \Omega_{a c} \Omega_{b d}
$$

While (14) was expected based upon earlier results in other supergravity theories [20], equation (17) was something of a surprise. Since the five-dimensional potential is invariant under the $S L(2, \mathbb{R})$ it was natural to think of these scalars as representing the holographic duals of the gauge coupling and $\theta$-angle. This, however, is only correct at the UV fixed point where the five-dimensional $S L(2, \mathbb{R})$ represents the UV gauge couplings, which are true moduli of the theory. The ten-dimensional dilation/axion coset represents the gauge couplings as they flow, and mix with other couplings. The radial dependence thus gives the running gauge couplings on the brane. 
Equation (17) is thus important for several reasons. It represents an integrated version of the Callan-Symanzik equation, and for supersymmetric flows it must also contain an integrated NSVZ beta-function [15]. This result also represents a cautionary tale for brane-worlds: The relationship between the five-dimensional variables, and the ten-dimensional fields can be quite subtle and complicated. In particular, the tendimensional dilaton and axion can behave in a highly non-trivial manner while the five dimensional fields in the corresponding $S L(2, \mathbb{R})$ remain fixed. It is only by lifting to the ten-dimensional solution that such relationships become manifest, and it is the tendimensional string theory, and not the five-dimensional field theory, that represents the "controlling authority" in IIB holography.

Returning to the "super-QCD," or $\mathcal{N}=1^{*}$, flow described in the previous section, one finds that the lift to ten dimensions has a rich structure. To describe it in detail, I must first parametrize $S^{5}$ in a rather unusual manner. Consider $S^{5}$ as the unit sphere in $\mathbb{R}^{3} \times \mathbb{R}^{3}$ :

$$
\vec{u}^{2}+\vec{v}^{2}=1, \quad \vec{u}, \vec{v} \in \mathbb{R}^{3} .
$$

The $S O(3) \mathcal{R}$-symmetry invariance of the flows acts on $S^{5}$ by simultaneous rotation of $\vec{u}$ and $\vec{v}$. Using such a rotation, we can reduce $\vec{u}$ and $\vec{v}$ to the form:

$$
\vec{u}=\left(0,0, y_{1}\right), \quad \vec{v}=\left(0, y_{2}, y_{3}\right),
$$

where $\sum_{j} y_{j}^{2}=1$. Thus, the 5 -sphere may be generically thought of an $S O(3) \equiv \mathbb{R P}^{3}$ fiber over an $S^{2}$ base parametrized by $\vec{y}$. However, an $S O(3)$ rotation can negate any two Cartesian coordinates, and so the base is really $S^{2} /\left(\mathbb{Z}_{2} \times \mathbb{Z}_{2}\right)$, where we take $y_{1}, y_{2} \geq 0$. This represents a quarter of a sphere, or a disk. Note that the perimeter of this disk is characterized by points at which $\vec{u}$ and $\vec{v}$ are parallel. If $\vec{u}$ and $\vec{v}$ are indeed parallel, then the $S O(3)$ action degenerates: there is an $S O(2)$ subgroup that fixes $\vec{u}$ and $\vec{v}$, and the fiber is thus $S^{2}=S O(3) / S O(2)$. The geometric picture of $S^{5}$ is thus an $S O(3)$ fibration over a unit disk, where the fiber degenerates to an $S^{2}$ at the perimeter. It is covenient to parametrize the disk in terms of the $S O(3)$-invariants:

$$
w_{1}=2 \vec{u} \cdot \vec{u}-1, \quad w_{2}=2 \vec{u} \cdot \vec{v}, \quad \text { where } 0 \leq w_{1}^{2}+w_{2}^{2} \leq 1 .
$$

So far all I have done is find a complicated description of $S^{5}$, but this description is precisely adapted to the $\mathcal{N}=1^{*}$ flows.

\subsection{Flows with $\lambda<1$ : Seven-branes}

Flows with $\lambda<1$ (see (11),(12)) have the gaugino condensate, $\sigma$, flowing "slowly," and as we will see it is the chiral multiplet mass that dominates in the infra-red, and determines the structure of the core of the supergravity solution. As we saw in (13), the 5 -metric becomes singular as $t \rightarrow 1$. However, in this limit one has $d r \sim \frac{d t}{t}$ and $\Delta^{-\frac{2}{3}} \sim \frac{1}{1-t}$. When substituted into (14), the simple pole in $\Delta^{-\frac{2}{3}}$ cancels the zero in front of the brane metric in (13), and the apparent singularity in $\frac{d t^{2}}{t^{2}(1-t)}$ can be removed by a change of variables $\chi=(1-t)^{\frac{1}{2}}$. Finally, the $\mathbb{R P}^{3}$ fiber metric, $d \Omega_{3}^{2}$, in the $S^{5}$ 
gets a prefactor of $(1-t) \sim \chi^{2}$. Thus one finds that the ten-dimensional metric (14) is asymptotic to:

$$
\begin{gathered}
d s^{2}=\frac{1}{\sqrt{2}} \widehat{\Omega}^{\frac{1}{4}}\left[2\left(1-\lambda^{2}\right)^{1 / 3} e^{2 C_{1}}\left(\eta_{\mu \nu} d x^{\mu} d x^{\nu}\right)-d \chi^{2}-\frac{1}{4} \chi^{2}\left(\sigma_{1}^{2}+\sigma_{2}^{2}+\sigma_{3}^{2}\right)\right] \\
-\frac{1}{\sqrt{2}} \widehat{\Omega}^{-\frac{3}{4}}\left[\frac{2(1-\lambda)}{(1+\lambda)} d w_{1}^{2}+\frac{(1+\lambda)}{2(1-\lambda)} d w_{2}^{2}\right]
\end{gathered}
$$

where

$$
\widehat{\Omega}=\left(1-w_{1}^{2}-w_{2}^{2}\right) .
$$

In taking this limit I have assumed that $(1-t)$ is small compared to $1-w_{1}^{2}-w_{2}^{2}$.

Observe that this metric is regular, except on the ring $w_{1}^{2}+w_{2}^{2}=1$. Moreover it consists of a flat $(7,1)$-metric and flat Euclidean 2-metric with warp-factors that are precisely appropriate to the reduction of the ten-dimensional theory down to eight dimensions. The throat of the D3-brane metric is therefore rounding out into a 7-brane world at $t \sim 1$, or at $r \sim C_{1}$. From (11) we have $m \sim \sqrt{3} e^{C_{1}} e^{-r}$ as $r \rightarrow \infty$, and hence the value of $r$ at which the throat rounds out is set by the logarithm of the UV mass scale. Also note that the scale on the $D 3$ brane limits to a finite value set by the UV mass scale $\left(e^{C_{1}}\right)$ and by $(\lambda-1)$. This suggests that this family of flows cannot access the far infra-red, and that the chiral multiplets do not fully decouple. It also suggests that the flows for $\lambda=1$ should be qualitatively different in this respect.

It is also amusing to note that the $(7,1)$-metric in (21) is actually not $\mathbb{R}^{7,1}$ but $\mathbb{R}^{3,1} \times \mathbb{R}^{4} / \mathbb{Z}_{2}$. This is because $d \Omega_{3}^{2}$ is the metric on $\mathbb{R} \mathbb{P}^{3}$ and not $S^{3}$. The presence of this $A_{1}$ singularity might well prove interesting in the string theory.

Finally, the dilaton background also develops a ring singularity that supports the 7-brane picture:

$$
\mathcal{S} \mathcal{S}^{T} \sim \widehat{\Omega}^{-\frac{1}{2}}\left(\begin{array}{cc}
1+w_{1} & w_{2} \\
w_{2} & 1-w_{1}
\end{array}\right)
$$

\subsection{The ring singularity: Duality-averaged branes}

In the foregoing I considered a limit in which $(1-t)$ was small compared to $1-w_{1}^{2}-w_{2}^{2}$. It is instructive to consider the opposite order of limits since it probes the ring singularity more closely. In particular, setting $w_{1}=\cos (2 \theta), w_{2}=\sin (2 \theta) \cos \phi$, one finds an asymptotic dilaton configuration of the form:

$$
\mathcal{S S}^{T} \sim Q \cdot\left(\begin{array}{cc}
\mathcal{U} \frac{1}{\sqrt{1-t}} & 0 \\
0 & \mathcal{U}^{-1} \sqrt{1-t}
\end{array}\right) \cdot Q^{-1}
$$

where

$$
\mathcal{U} \equiv\left(\frac{2\left(1-\lambda^{2}\right)}{1+2 \lambda \cos (4 \theta)+\lambda^{2}}\right)^{\frac{1}{2}}, \quad Q \equiv\left(\begin{array}{cc}
\cos \theta & -\sin \theta \\
\sin \theta & \cos \theta
\end{array}\right) .
$$

In terms of the IIB coupling, $\tau$ one has:

$$
\tau=\frac{i \mathcal{U} \cos \theta-\sqrt{1-t} \sin \theta}{\sqrt{1-t} \cos \theta+i \mathcal{U} \sin \theta} \sim \cot \theta \quad \text { as } t \rightarrow 1 \text {. }
$$


Observe that the singularity structure depends only rather weakly upon $\lambda$, and that, apart from a varying, but positive normalization factor, the ring singularity appears to be rotationally invariant.

Recall that for $\lambda=0$ the flow has an $S O(3) \times S O(2)$ symmetry, and that the $S O(2)$ symmetry is a combination of a duality rotation and the geometric rotation of $\vec{u}$ into $\vec{v}$. Equation (24) shows that the ring singularity is generated by precisely such a symmetry: the singularity is smeared into the ring with a simultaneous duality rotation. Moreover (26) shows that $\tau$ approaches the real axis as $t \rightarrow 1$ : At finite $N$, a singularity at $\operatorname{Im}(\tau)=0$ can be interpreted in terms $(p, q)$-branes provided that $\tau$ approaches a rational point on the real axis. In the limit $N \rightarrow \infty$ one gets a smooth distribution $(p, q)$-branes. While this $S O(2)$ rotation is only a symetry for $\lambda=0$, one sees that for $0<\lambda<1$ the picture is qualitatively the same, and thus the infra-red structure is primarily determined by the flow of the chiral multiplet mass. As we will see, the structure is rather different for $\lambda=1$.

\subsection{Flows with $\lambda=1$ : Five-branes}

If one looks at (21) one sees that various coefficients either vanish or diverge as $\lambda \rightarrow 1$. Re-examining the asymptotics of the ten-dimensional metric for $\lambda=1$ yields:

$$
d s^{2} \sim \widetilde{\Omega}^{\frac{1}{4}}\left[2^{\frac{2}{3}} 3^{\frac{1}{3}} e^{2 C_{1}} \chi^{\frac{2}{3}}\left(\eta_{\mu \nu} d x^{\mu} d x^{\nu}\right)-d \chi^{2}\right]-\widetilde{\Omega}^{-\frac{3}{4}}\left[\frac{1}{3 \chi^{2}} d w_{2}^{2}+d \tilde{s}_{4}^{2}\right],(
$$

where $d \tilde{s}_{4}^{2}$ is a complicated, but regular metric on $\mathbb{R I P}^{3}$ and in the $\theta$ direction. The warp factor, $\widetilde{\Omega}$, is now given by:

$$
\widetilde{\Omega} \equiv \frac{1}{9}\left(3 \cos ^{2}(2 \theta)+4 \sin ^{2}(2 \theta) \sin ^{2}(\phi)\right) .
$$

The dilaton matrix takes the form

$$
\mathcal{S} \mathcal{S}^{T} \sim \frac{1}{3} \widetilde{\Omega}^{-\frac{1}{2}}\left(\begin{array}{cc}
1+2 \cos ^{2}(\theta) & 2 \sin (2 \theta) \cos \phi \\
2 \sin (2 \theta) \cos \phi & 1+2 \sin ^{2}(\theta)
\end{array}\right) .
$$

The metric and the dilaton no longer have a ring singularity, but only have a singularity at the points $\theta= \pm \frac{\pi}{4}, \phi=0$. On the other hand, the metric now has a singularity at $\chi=0$. It is not so simple to give this metric a geometric interpretation, particularly since one of the internal directions is blowing up as $\chi \rightarrow 0$. Also note that the coefficient of the D3-brane metric vanishes as $\chi \rightarrow 0$, which, in principle, suggests that the flow probes arbitrarily far into the infra-red.

The metric and dilaton are actually a little more regular near $\chi=0, \theta= \pm \pi / 4$,

$\phi=0$, than is apparent from the foregoing. Setting $\theta=\frac{\pi}{4}+\psi, t=1-\frac{1}{2} \chi^{2}$ and expanding in small $\chi, \psi$ and $\phi$ one finds:

$$
\begin{aligned}
d s^{2} \sim 2 \widetilde{\Omega}^{\frac{1}{4}}\left[3^{\frac{1}{3}} e^{2 C_{1}} \chi^{\frac{2}{3}}\left(\eta_{\mu \nu} d x^{\mu} d x^{\nu}\right)-d \chi^{2}\right] & -\frac{1}{2 \sqrt{3}} \widetilde{\Omega}^{-\frac{3}{4}} \chi^{2}\left[\frac{16}{3} d \psi^{2}+d \phi^{2}+\phi^{2}\left(\sigma_{1}^{2}+\sigma_{2}^{2}+\sigma_{3}^{2}\right)\right]
\end{aligned}
$$

where one now has:

$$
\widetilde{\Omega} \equiv \frac{4}{9}\left(3 \psi^{2}+\phi^{2}+\chi^{4}\right) .
$$


Note that the metric $(30)$ has round $\mathbb{R P}^{3}$ fibers, but there is a conical singularity at $\phi=0$. The dilaton matrix becomes:

$$
\mathcal{S} \mathcal{S}^{T} \sim \frac{2}{3} \widetilde{\Omega}^{-\frac{1}{2}} \mathcal{Q}\left(\begin{array}{cc}
2 & -\psi \\
-\psi & 2 \psi^{2}+\frac{1}{2} \phi^{2}+\frac{1}{2} \chi^{4}
\end{array}\right) \mathcal{Q}^{T},
$$

where $\mathcal{Q}$ is a rotation by $\theta=\pi / 4$.

Motivated by [12] one would like to interpret this solution in terms of 5-branes. Indeed, it is apparent from the dilaton configuration that the foregoing solution appears to represent an S-dual pair of 5-branes: one of type $(1,1)$ and the other of type $(1,-1)$. The metric contains an $S O(4)$-invariant sector which presumably represents the 3 -spheres (or at least $\mathbb{R P}^{3}$ 's) transverse to the putative 5 -branes.

\section{Supergravity and the phases of super QCD}

The vacua of the $\mathcal{N}=1^{*}$ theories have been extensively studied [21, 22]. The basic point is that the chiral multiplet mass modifies the superpotential so that the ground-state vevs for the (complex) scalars must satisfy:

$$
\left[\Phi_{i}, \Phi_{j}\right]=-\frac{m}{\sqrt{2}} \epsilon_{i j k} \Phi_{k} .
$$

If the mass parameter, $m$, is real then the solution to this equation is to take the $\Phi_{j}$ to be some real combination of the anti-hermitian generators of an $S U(2)$ subgroup of $S U(N)$. It was thus argued in 113 , 12] that the ground states of the $\mathcal{N}=1$ theory should correspond to the $D 3$-branes becoming dielectric 5-branes that wrap a noncommutative $S^{2}$ whose radius is determined by $\sum_{j} \operatorname{Tr}\left(\left|\Phi_{j}\right|^{2}\right) \sim m^{2} C_{2}(R)$, where $C_{2}(R)$ is the quadratic Casimir of the $S U(2)$ representation, $R$, induced by the embedding of $S U(2)$ into $S U(N)$.

To relate the field theory to the supergravity flows, recall that for finite $N$ and for commuting vevs, the $\Phi_{j}$ may be thought of as the Cartesian coordinates transverse to the $D 3$-branes. More precisely, the solutions here have an $S O(3)$-invariance: In (33) the (real) $S O(3)$ acts the indices $i, j, k$, with the real and imaginary parts of $\Phi_{i}$ transforming separately, each as a triplet of $S O(3)$. Thus the real and imaginary parts of $\vec{\Phi}$ correspond to the coordinates $\vec{u}$ and $\vec{v}$ on $S^{5}$. If we were to obtain precisely the solution of [12] then the 5-branes should emerge in the limit in which $v_{j} \equiv 0$ : Instead we find (for $\lambda<1$ ) a ring singularity when $\vec{u}$ and $\vec{v}$ are parallel. The reason for this difference is easily understood: the supergravity solution has an extra continuous duality symmetry, and for $\lambda<1$ the solution is smeared out into a "duality averaged" family of type $(p, q)$ branes. This continuous symmetry must be broken by the string theory, or for finite $N$ field theory, and presumably the ring singularity must resolve itself into a discrete rational family of type $(p, q)$ branes.

For $\lambda=1$, one arrives at something much closer to the ideas of [13, [12]. In

particular, the supergravity solution has symmetries that are consistent with string theory, and finite $N$ field theory, and appear to represent a pair of $S$-dual, oblique confinement phases of the field theory. 
The flows with $\lambda<1$ also exhibit the interesting new feature of "rounding out" into a 7-brane world of finite scale except when the vevs of $\vec{\Phi}$ satisfy the reality condition $(\vec{u}, \vec{v}$ parallel) for which there is a vacuum state in the $\mathcal{N}=1^{*}$ theory. Thus the supergravity theory "knows" that there is no $(3,1)$-dimensional infra-red, field theory limit below the energy scale of the chiral multiplet mass, unless the vevs obey the proper reality condition.

\section{Final comments}

From the detailed analysis of the $\mathcal{N}=1^{*}$ flows it is clear that working purely in five dimensions conceals much that is important. The details of how the flows behave in the far infra-red only become apparent once one lifts to ten dimensions. Because of the warp-factors, apparently singular metrics in five dimensions become far more regular in ten dimensions, and the singularities of the ten-dimensional solutions develop a rich but interpretable structure. In addition, contrary to naive expectations, the dilaton and axion flow in the ten-dimensional theory in a manner that is entirely determined by the running of the masses of the chiral multiplets and the gaugino vevs. Apart from giving information about the running coupling on the brane, the behaviour of the dilaton and axion also gives important information about the types of branes that emerge in the infra-red. While these details are, in principle, encrypted in the five-dimensional solution, the only way to make them apparent is to construct the lift to ten dimensions.

The supergravity solutions discussed here mesh very nicely with one's expectations based upon field theory. It is interesting to see how the supergravity solution "roundsout" so as to prevent access to the infra-red when the scalar vevs do not satisfy the proper reality conditions. One also sees the emergence of various types of dielectric 5-brane singularities and, depending on how the gaugino vev flows, one sees how the symmetries of the five-dimensional flow act on the 5-brane sources to produce a ring singularity, or a discrete pair of singularities.

Another lesson coming from the supergravity solutions described here is that the details of the infra-red singularity can depend discontinuously upon vevs: Here the infrared limit was essentially the same for all $\lambda<1$, but changed dramatically at $\lambda=1$. This suggests that in the infra-red there are only a discrete set of choices for vevs, and that the corresponding choices in the UV dramatically change the infra-red physics. This is precisely what is found in the field theory [22].

This points to a draw-back of using $\mathcal{N}=8$ supergravity: It requires that one restrict

ones attention to a very special sub-class of possible excitations. One has thus made some implicit choices of vevs in the UV, and so it will restrict the class IR fixed point theories. In particular, $\mathcal{N}=8$ supergravity methods can see only a limited subset of the general family of fixed points outlined in [12, 21, 22]. To access the broader classes of fixed points one must allow several different operator vevs to flow independently, and thus one must go back to the full ten-dimensional theory.

Thus, five-dimensional supergravity methods are very powerful in generating exact 
solutions, whereas competing ten-dimensional approaches 12 usually have to resort to linear approximations. Moreover, five-dimensional methods have led to significant insights into holographic field theories. However, the five-dimensional methods have limitations: There are restrictions on the families of solutions, and there is the "myopia" of the five-dimensional perspective. It has been shown here that at least the myopia can be completely corrected by lifting to ten dimensions.

Before concluding I wish to try to extract a further lesson from the $\mathcal{N}=1^{*}$ flows considered here. It was shown that the radius at which the brane-world geometry rounds out is given by $r_{0} \sim \log \left(m_{U V}\right)$, where $m_{U V}$ is the UV mass parameter that induces the breaking of $\mathcal{N}=4$ to $\mathcal{N}=1$ Yang-Mills. Such logarithms are very familiar in brane-world scenarios, and indeed one of the attractive features of these scenarios is that large energy scale differences are obtained from small geometric separations precisely through such logarithms. However, in the supergravity solution presented here, the brane geometry is not something that is chosen: It is a derived property from the "initial datum," $m_{U V}$. Thus the freely choosable parameter still suffers from the hierarchy problem, and it raises the issue as to whether brane-world scenarios actually provide a solution to the hierarchy problem, or merely yield a geometric encoding of a logarithm.

Whatever conclusion one makes about brane-worlds and the hierarchy problem, the work described here illustrates a rather more general cautionary tale: To properly interpret a flow involving brane-world physics one must embed the branes in the full, parent string theory

\section{Acknowledgments}

I would particularly like to thank my collaborator, Krzysztof Pilch: The results presented here are based largely upon our joint work. I am also very grateful to the organizers of the Gürsey Memorial Conference for opportunity to present this material, and to the Aspen Center for Physics for its hospitality while I clarified some of the issues underlying this work. This work was supported in part by funds provided by the DOE under grant number DE-FG03-84ER-40168.

\section{References}

[1] J. Maldacena, The Large N Limit of Superconformal Field Theories and Supergravity, Adv. Theor. Math. Phys. 2 (1998) 231; hep-th/9711200.

[2] S.S. Gubser, I.R. Klebanov, A.M. Polyakov, Gauge Theory Correlators from Non-Critical String Theory, Phys. Lett. B428 (1998) 105, hep-th/9802109;

E. Witten, Anti-de Sitter Space and Holography, Adv. Theor. Math. Phys. 2 (1998) 253, hep-th/9802150.

[3] L. Girardello, M. Petrini, M. Porrati and A. Zaffaroni, Novel Local CFT and Exact Results on Perturbations of $N=4$ Super Yang-Mills from AdS Dynamics, J. High Energy Phys. 12 (1998) 022, hep-th/9810126; 
J. Distler and F. Zamora, Nonsupersymmetric Conformal Field Theories from Stable Anti-de Sitter Spaces, Adv. Theor. Math. Phys. 2 (1999) 1405, hep-th/9810206.

[4] D. Z. Freedman, S. S. Gubser, K. Pilch, and N. P. Warner, Renormalization Group Flows from Holography-Supersymmetry and a c-Theorem, CERN-TH-99-86, ; to appear in Adv. Theor. Math. Phys. $\underline{3}$ (2000); hep-th/9904017.

[5] V. Novikov, M. A. Shifman, A. I. Vainshtein, V. Zakharov, Exact Gell-Mann-Low Function of Supersymmetric Yang-Mills Theories from Instanton Calculus, Nucl. Phys. B229 (1983) 381;

I. I. Kogan, M. A. Shifman, and A. I. Vainshtein, Matching Conditions and Duality in N=1 SUSY Gauge Theories in the Conformal Window, Phys. Rev. D53 (1996) 4526, hep-th/9507170.

[6] A. Khavaev, K. Pilch and N.P. Warner, New Vacua of Gauged N=8 Supergravity in Five Dimensions, Phys. Lett. $\underline{B 487}$ (2000) 14; hep-th/9812035.

[7] D. Z. Freedman, S. S. Gubser, K. Pilch, and N. P. Warner, Continuous Distribution of D3branes and Gauged Supergravity, J. High Energy Phys. $\mathbf{7}$ (2000) 38; hep-th/9906194.

[8] L. Girardello, M. Petrini, M. Porrati and A. Zaffaroni The Supergravity Dual of $\mathcal{N}=1$ Super-Yang-Mills Theory, Nucl. Phys. B569 (2000) 451, hep-th/9909047.

[9] K. Behrndt, Domain walls of $D=5$ supergravity and Fixed Points of $\mathcal{N}=1$ Super YangMills, hep-th/9907070.

[10] M. Petrini and A. Zaffaroni, The Holographic RG Flow to Conformal and Non-Conformal Theory, hep-th/0002172.

[11] N. Evans and M. Petrini, AdS RG Flow and the Super-Yang-Mills Cascade, SHEP-00-05, IMPERIAL-TP-99-00-28, hep-th/0006048.

[12] J. Polchinski and M. J. Strassler, The String Dual of a Confining Four-Dimensional Gauge Theory, hep-th/0003136.

[13] R.C. Myers, Di-electric Branes, J. High Energy Phys. 9912 (1999) 22; hep-th/9910053.

[14] K. Pilch and N.P. Warner, A New Supersymmetric Compactification of Chiral, IIB Supergravity, CITUSC/00-012, USC-00/01; Phys. Lett. $\underline{B 487}$ (2000) 22; hep-th/0002192.

[15] K. Pilch and N.P. Warner, $\mathcal{N}=2$ Supersymmetric $R G$ Flows and the IIB Dilaton, CITUSC/00-18, USC-00/02; hep-th/0004063.

[16] K. Pilch and N.P. Warner, $\mathcal{N}=1$ Supersymmetric Renormalization Group Flows from IIB Supergravity, CITUSC/00-024, USC-00/03, hep-th/0006066.

[17] L. Randall and R. Sundrum, A Large Mass Hiearchy from a Small Extra Dimension, Phys. Rev. Lett. $\underline{83}$ (1999) 3370, hep-ph/9905221; An Alternative to Compactification, Phys. Rev. Lett. $\underline{83}$ (1999) 4690, hep-th/9906064;

[18] J. de Boer, E. Verlinde and H. Verlinde, On the Holographic Renormalization Group, J. High Energy Phys. $\underline{\mathbf{8}}$ (2000) 3; hep-th/9912012.

[19] M. Günaydin, L.J. Romans and N.P. Warner, Gauged $N=8$ Supergravity in Five Dimensions, Phys. Lett. 154B (1985) 268; Compact and Non-Compact Gauged Supergravity Theories in Five Dimensions, Nucl. Phys. B272 (1986) 598.

M. Pernici, K. Pilch and P. van Nieuwenhuizen, Gauged $N=8 D=5$ Supergravity, Nucl. Phys. B259 (1985) 460.

[20] B. de Wit and H. Nicolai, On the Relation Between $d=4$ and $d=11$ Supergravity, Nucl. Phys. B243 (1984) 91;

B. de Wit, H. Nicolai and N.P. Warner, The Embedding of Gauged $N=8$ Supergravity into $d=11$ Supergravity, Nucl. Phys. B255 (1985) 29.

[21] C. Vafa and E. Witten, A Strong Coupling Test of S Duality, Nucl. Phys. B431 (1994) 
3 , hep-th/9408074;

R. Donagi and E. Witten, Supersymmetric Yang-Mills Theory and Integrable Systems, Nucl. Phys. B460 (1996) 299; hep-th/9510101.

[22] N. Dorey, An Elliptic Superpotential for Softly Broken $\mathcal{N}=4$ Supersymmetric Yang-Mills Theory, J. High Energy Phys. 9907 (1999) 021; hep-th/9906011.

N. Dorey and S. Prem Kumar, Softly-Broken $\mathcal{N}=4$ Supersymmetry in the Large- $N$ Limit, J. High Energy Phys. 0002 (2000) 006, hep-th/0001103;

O. Aharony, N. Dorey and S. Prem Kumar, New Modular Invariance in the $\mathcal{N}=1^{*}$ Theory, Operator Mixings and Supergravity Singularities, hep-th/0006008. 